\title{
Operations optimization of memory polynomial with binomial reduction in digital pre- distortion for wireless communication systems
}

\begin{abstract}
The non-linear characteristic of the Power Amplifier (PA) causes system inefficiency and signal distortion when operating in the non-linear region. High Peak to Average Power Ratio (PAPR) in recent high speed wireless communications technology causes Memory Effects, where the PA output signal deteriorates with unwanted scattering and distortion against the ideal signal value. To solve the PA non-linearity effects, Digital Pre-distortion (DPD) is chosen among other linearization methods due to its attractive strengths on ease of implementation, supported bandwidth, flexibility, efficiency and cost. A precise modeling of the PA is required in order to compliment the effectiveness of DPD in terms of resources and performance. Memory Polynomial (MP) has been employed widely in the industry and academia on PA modeling due to its significant resource reduction from Volterra Series. The Memory Polynomial with Binomial Reduction method (MPB-imag-2k) was developed where similar performance is achieved using lesser resources compared to MP. MPB-imag-2k was enhanced into MPB on its Normalized Mean Square Error (NMSE). This paper exhibits the resource optimization in terms of calculation and multiplication operations by using the Calculation Complexity Reduction Ratio (CCRR). CCRR is derived into Multiplications Operations Reduction Ratio (MORR) together with Addition Operations Reduction Ratio (aORR) for both MPB and MP. A modeled ZVE-8G PA and sampled 4G (LTE) signals are used in the simulation. MPB is compared with MP in the non-linearity order range of 1 to 4, pre-amplifier gain (PAG) of 2 to 4, with up to 36dB improvement in NMSE, 57\% of MORR and $87.5 \%$ of AORR.
\end{abstract}

Keyword: Power amplifier; PA linearization; Digital pre-distortion; 4G; Memory polynomial 